\title{
LENTES DOS VALORES E PRINCÍPIOS DA PROMOÇÃO DA SAÚDE SOBRE A COVID-19
}

LENSES OF VALUES AND PRINCIPLES FOR HEALTH PROMOTION REGARDING COVID-19

LENTES DE LOS VALORES Y PRINCIPIOS DE PROMOCIÓN DE LA SALUD ACERCA DEL COVID-19

\section{Como Citar:}

Dias MSA. Lentes dos valores e princípios da promoção da saúde sobre a covid-19. Sanare (Sobral, Online). 2021; 20(2):72-78.

Descritores:

Promoção da Saúde; Pandemias; covid-19.

Descriptors:

Health promotion; Pandemics;

Covid-19.

Descriptores:

Promoción de la Salud; Pandemias; covid-19.

Submetido: $17 / 09 / 2021$

Aprovado:

$17 / 11 / 2021$

Autor(a) para Correspondência: Maria Socorro de Araújo Dias Universidade Estadual Vale do Acaraú

Rua Maestro José Pedro, 170 Centro, Sobral - CE, 62010-260 E-mail: socorroad@gmail.com

\section{RESUMO}

Este ensaio teórico desenvolve um argumento: os valores e princípios da promoção da saúde demarcados na Política Nacional de Promoção da Saúde e sua pertinência para um agir ético-político apresentam potencial contributivo para a indução e implementação de medidas de proteção social por meio de políticas públicas, de produção de saberes e práticas com participação da sociedade civil, que visam colaborar na efetivação e na tomada de decisão em saúde ancoradas no reconhecimento dos múltiplos determinantes do processo de produção de saúde e de vida, com efeitos no contexto pandêmico da covid-19 e de seus desdobramentos no curso do pós-pandemia.
1. Enfermeira. Doutora em Enfermagem, com Pós-doutoramento em Cuidados Clínicos em Enfermagem e Saúde. Universidade Estadual Vale do Acaraú. E-mail: socorroad@gmail.com. ORCID: https://orcid.org/0000-0002-7813$\underline{547 X}$

Cert. de Redação Científica: Central das Revisões. Edição de texto: Karina Maria M. Machado. Revisão de provas: Texto definitivo validado pelos (as) autores(as). 


\section{ABSTRACT}

This theoretical essay develops an argument: the values and principles for health promotion outlined in the National Policy for Health Promotion, and their relevance to an ethical-political act, have the potential to contribute in inducing and implementing social protection measures by means of public policies, for the production of knowledge and practices with the participation of civil society, which aim to collaborate in realizing and making health-related decisions, based on the recognition of the multiple determinants of the life and health production process, with effects in the pandemic context of covid-19 and its consequences in the post-pandemic course.

\section{RESUMEN}

Este ensayo teórico desarrolla un argumento: los valores y principios de la promoción de la salud demarcados en la Política Nacional de Promoción de la Salud y su relevancia para un actuar ético-política presentan potencial de contribución a la inducción e implementación de medidas de protección social a través de políticas públicas, de producción de saberes y prácticas con participación de la sociedad civil que tienen como objetivo colaborar en la implementación y tomada de decisiones en salud, anclado en el reconocimiento de los múltiples determinantes del proceso de producción de vida y salud con efectos en el contexto pandémico de CoVID-19 y su evolución en el curso de la postpandemia.

\section{INTRODUÇÃ O}

$\mathrm{Na}$ história moderna, nenhuma outra situação de saúde teve tanto impacto mundial e desafiou cosmovisões de sociedade saudável como a vivenciada pela covid-191. 0 mundo vive a maior crise sanitária deste século. Alinhada a esta, temse o reconhecimento de uma conjuntura política e econômica ancorada na defesa intransigente do capital que acentua políticas de austeridade e exacerba as desigualdades sociais. Este contexto requer mobilidade de todos para a adoção e/ ou reinvenção de valores na tomada de decisões socialmente responsáveis.

Uma crise sanitária produz repercussões em escala global e tem como consequências imediatas a perda de vidas ou o agravamento de condições de adoecimento prévio. Ademais, agravam-se os impactos não somente de cunho epidemiológico, há evidências de crescentes repercussões políticas, econômicas e sociais na vida humana. Pessoas e comunidades padecem com os efeitos da covid-19.

A pandemia ocasionada pelo SARS-CoV-2 mostrou que os avanços na luta contra a pobreza e a fome são insuficientes, acentuou a visibilidade da intrínseca relação corpo-mente e desnudou o potencial maléfico, multideterminado e determinante das desigualdades sociais na vida em sociedade.

0 enfrentamento da covid-19 e de suas repercussões requer dos governantes tomar medidas urgentes e corajosas². Além disso, fazem-se necessários sólidos embasamentos científicos e diretrizes operacionais que subsidiem 0 desenvolvimento de ações impostergáveis para melhor lidar com os múltiplos desafios originados ou potencializados por esta pandemia, para os quais a promoção da saúde tem acúmulo para induzir e produzir respostas "por meio de uma abordagem integrativa"1.

No Brasil, a Política Nacional de Promoção da Saúde (PNPS), instituída em 2006, passou por processo de atualização em 2014. Nesta, a conceitualização de Promoção da Saúde se vincula coerentemente ao conceito ampliado de saúde e sinaliza que sua materialização dar-se-á por meio de estratégias para a produção de saúde individual e coletiva, a partir de articulações e cooperações intra e intersetorial, pela formação de redes de atenção à saúde e de proteção social. Enfatiza ainda a necessária participação social neste processo $0^{3,4}$.

Ademais, apresenta valores e principios indutores para o campo de práticas da promoção da saúde. Estes fundam-se na premissa máxima de defesa da saúde e da vida, reconhecendo o processo de subjetivação de pessoas e coletivos 4 .

Com base nessas considerações iniciais, admitese, neste ensaio, que os princípios e valores da promoção da saúde expressos na PNPS apresentam potencial indutor para a formulação e implementação de políticas públicas para o enfrentamento das desigualdades potencializadas e desnudadas no contexto da covid-19 e seus efeitos previsíveis no curso do pós-pandemia.

\section{Política e Saúde: é possível dissociá-las?}

A pandemia de covid-19 sugere que não. Aliás, 
essa máxima - associação entre política e saúde já é reconhecida. Portanto, a premissa verdadeira é: não podemos dissociar a saúde da política. É na sinergia entre política e saúde ou na ausência dela que se instauram em maior ou menor proporção os efeitos na saúde pública. Similarmente, políticas públicas podem ser intensamente afetadas por eventos adversos na saúde.

A crise econômica global foi profundamente intensificada pela circulação do SARS-CoV-2, as taxas de desemprego aumentaram e a insegurança social se instaurou. Contudo, é preciso reconhecer que “as pandemias raramente afetam todas as pessoas de maneira uniforme" ${ }^{5}$. As taxas de mortalidade são desproporcionais entre os extratos sociais, afetando acentuadamente os pobres e os desfavorecidos. Mitigar os efeitos devastadores da atual pandemia requer tomar em consideração o impacto dos determinantes sociais da saúde e reconhecer a necessidade de políticas intersetoriais coordenadas.

No Brasil, a crise sanitária se conjugou às crises política e econômica. É no contexto desta tríade que, em fevereiro de 2020, o Brasil teve seu primeiro caso confirmado. Sete meses depois, o país registrou, oficialmente, 4.624 .885 casos e 138.997 óbitos (em 23 de setembro de 2020) ${ }^{6}$. Em uma análise espacial entre as unidades federativas, todos os 23 estados e o Distrito Federal registraram indicadores que denotam a acentuação da doença em nosso país, embora com variação temporal nas curvas epidemiológicas.

Marcada por antecedentes históricos de pobreza estrutural indutora de desigualdades sociais, a população brasileira tem enfrentado os efeitos consideráveis destes nos indicadores epidemiológicos relativos à covid-19 e em suas repercussões socioeconômicas e culturais. Concernente a este pensamento, autores ${ }^{7}$ advertem que embora tenha sido atribuído à pandemia em curso um efeito equalizador ao requerer de todos medidas de distanciamento físico, ficou evidente que os determinantes sociais da saúde acentuam profunda e desigualmente a morbidade e mortalidade relacionada à covid-19, sendo consistente esta associação.

No Brasil, o Plano Nacional de Enfrentamento à pandemia de covid-19, elaborado por um conjunto de organizações que atuam no campo da saúde, cunhou que os desafios para o enfrentamento da covid-19 no país são mais contundentes dada a complexidade de grande desigualdade econômica e social. Outrossim, reconhece que o controle da pandemia e a redução dos seus impactos sociais e sanitários requerem políticas que priorizem as vidas com proteção social em detrimento dos lucros ${ }^{8}$.

Este país extremamente desigual dispõe de um sistema de saúde que tem em seu bojo os princípios da universalidade e da igualdade, lido por muitos sanitaristas como equidade. É o Sistema Único de Saúde (SUS) que busca, cotidianamente, responder, sistemicamente, da Atenção Primária à oferta de atenção à saúde em contexto hospitalar ultraespecializado, às conhecidas e novas necessidades de saúde da população.

No curso da pandemia, o SUS e suas políticas estão sob análise. Dentre estas, a PNPS. Em evento organizado pela Associação Brasileira de Saúde Coletiva, por meio do Projeto Ágora, foram discutidas “As contribuições do campo da Promoção da Saúde para o enfrentamento da pandemia"1. Neste colóquio virtual, problematizou-se sobre as contribuições do campo da Promoção da Saúde para a pandemia e vice-versa. Acenou-se à convergência e pertinência da promoção da saúde enquanto referencial teóricometodológico e, no caso brasileiro, como política pública para a análise meticulosa das repercussões da pandemia de covid-19 e de suas consequências, partindo-se do entendimento de que a pandemia não é problema exclusivo do setor saúde, e sim de toda a esfera governamental, incluindo a econômica, e da sociedade em sua pluralidade. 0 debate também foi permeado pela afirmação da necessidade de sustentabilidade do SUS como política de Estado, inclusiva e universal.

Ademais, Marcos Akerman abordou a necessidade de engajamento da população e de uma boa governança como postura política para não deixar “o cavalo de Tróia invadir". Regiane Rezende apregoou que "a geografia da desigualdade se acentua cada vez" no contexto da pandemia e que é necessário por em cena os ensinamentos da Promoção da Saúde; Samuel Moyses advertiu para as consequências do ato humano, por vezes predatório, e que conduzem a tragédias humanas, para as quais a promoção da saúde tem discutido e apontado caminhos. Socorro Dias afirmou que: "A promoção da saúde tem muito a dizer para o enfrentamento da pandemia causada

1. Colóquio realizado em 18 de junho de 2020, que contou com a participação dos professores Marco Akerman (ESP-USP), Regiane Rezende (OPAS/OMS), Samuel Moysés (PUC PR) e Socorro Dias (UVA/ESP-VS), coordenado por Dais Rocha (UNB). Todos os integrantes do Grupo Temático de Promoção da Saúde e Desenvolvimento Local Integrado e Sustentável (GTPSD-ABRASCO). 
pelo novo coronavírus, porque produziu, desnudou e anuncia várias estratégias que visam à garantia da saúde e da vida como direito supremo". E indicou a defesa "quase intransigente" de sistemas de saúde universais e equânimes como reafirmação da saúde enquanto direito, admitindo ser inconcebível fazer a travessia da pandemia de covid-19 sem a presença do SUS ${ }^{9}$.

Estatísticas realçaram os efeitos consideráveis dos determinantes sociais como indutores de maior exposição ao vírus, atestados por estudos que demonstram que a taxa de infecção de covid-19 chega a ser três vezes maior em condados negros e/ou pobres do que em brancos. Quando se refere à mortalidade, a taxa passa a ser mais expressiva, alcançando seis vezes mais ${ }^{7}$.

Do exposto, deduz-se que a produção de saúde é multideterminada, requer ação política coordenada das várias instâncias de saberes e atuação e que essa associação está mais aclarada diante da pandemia. Outrossim, visualiza-se que a PNPS assume em seu texto essa compreensão e que tem o potencial de, enquanto política, por essência transversal às demais políticas, orientar e instrumentalizar processos e práticas que sejam convergentes com a defesa da vida, com o agir pautado na equidade e guiado pela busca da superação das desigualdades.

\section{Valores e princípios da Promoção da Saúde: Caixa de Ferramenta para mitigar iniquidades acentuadas pela covid-19}

Consideram-se como valores basilares da promoção da saúde: a solidariedade, a felicidade, a ética, o respeito às diversidades, a humanização, a corresponsabilidade, a justiça e a inclusão social ${ }^{4}$. Numa sociedade historicamente marcada pelo predomínio da exclusão social, potencializada por interesses individuais traduzidos em competitividade, o exercício da solicitude para com o outro, da repartição equitativa e da justiça com vistas à garantia de acesso aos benefícios da vida para todos, orientados para a redução das iniquidades, é um desafio a ser superado. Desafio este que se apresentou ainda mais expressivo no decorrer da pandemia.

0 curso da pandemia de covid-19 registrou, no Brasil, a luta pelo acesso à atenção à saúde especializada. Popularizou-se o termo "respirador" como dispositivo essencial à vida, e assim o é quando a condição clínica requer, a exemplo de muitos casos vividos por brasileiros - e no mundo inteiro. Entretanto, não parece ter sopesado o acesso, também, a insumos básicos para a manutenção mínima de um estado de higidez; visto que as recomendações necessárias para o contingenciamento do vírus requeriam como estratégia de mitigação do SARSCoV-2 o confinamento domiciliar, difícil de ser aderido por razões relativas às condições de moradias compartilhadas por mais de uma família, área física reduzida, urgência em se manter em atividades ainda quando não desejada e não permitida, além da população que vive em situação de rua. Abrams e Szefler ${ }^{7}$ reconhecem que os efeitos da pandemia de covid-19 permitiram lançar luz sobre as disparidades presentes nas sociedades contemporâneas, a exemplo dessas mencionadas.

É fato, porém, que se presenciou um apelo ao exercício da solidariedade e foram registrados muitos movimentos nessa procura. Situações que mobilizaram pessoas da comunidade e grandes empresas. Ousa-se dizer que, em história recente, não se reconhecem movimentos similares; o que não o torna suficiente, porém, denota uma chama acesa a aclarar esse valor nas relações humanas. Contudo, com os movimentos de retomadas das atividades "cotidianas", os sinais aclarados por alguns para o exercício dessa solicitude humana parecem desvairar. Solidariedade é grafada na PNPS como "razões que fazem sujeitos e coletivos nutrirem solicitude para com o próximo, nos momentos de divergência ou dificuldades" 4 . Definição que expressa em sua intencionalidade plena pertinência à experiência da pandemia em curso, mas que não pode limitar-se a esta. Ademais, há uma falsa sinalização de fim da pandemia, e torna-se importante elucidar que esta não finalizará com a diminuição ou ausência da circulação do vírus, pois suas consequências sociais perdurarão por período indefinido.

Outrossim, faz-se mister tencionar como esses valores expressos na PNPS são incorporados nas políticas públicas e na formação de cidadãos. A exemplo da solidariedade, a justiça e inclusão social, a corresponsabilização e o agir ético pressupõem condutas, ações e intervenções sustentadas pela valorização e defesa da vida, pautadas para o bem comum. Valores expressos na PNPS e que devem ser requeridos aos tomadores de decisões e, em especial, acionados pelos gestores no decorrer da pandemia, orientando uma postura de liderança ativa com capacidade de agregar instituições, pessoas e processos com vistas a mitigar os danos 
desta emergência sanitária. Sucede, no entanto, que foram presenciadas posturas díspares, gestores que se posicionaram e agiram na defesa intransigente da vida, diante do dilema da manutenção do isolamento como estratégia de controle da disseminação do vírus e proteção à vida. E outros que subestimaram as consequências e indicadores epidemiológicos e se posicionaram favoráveis à reabertura da economia, com posturas, muitas vezes, que revelavam a normalização da morte e negacionismo da ciência. 0 Estado não pode negligenciar o seu papel.

0 coletivo de associações na Frente pela Vida, ao sistematizar o Plano Nacional de Enfrentamento da pandemia de covid-198, cunhou a necessidade de descolonizar este modo de pensar e de agir com fulcro na supremacia patriarcal e capitalista e avançar para a construção de novas narrativas com valorização da Ciência e de outros conhecimentos produzidos por experiências e linguagens advindas dos movimentos sociais. Ademais, é requerido do Estado o protagonismo no enfrentamento do controle e consequências oriundas ou agravadas pela pandemia.

Em recente editorial, Buss e Belfort Jr. ${ }^{10}$ reconhecem a importância do auxílio emergencial, enquanto estratégia em curto prazo, por parte do Estado brasileiro, como medida que proporcionou alívio às pessoas mais despossuídas. Outrossim, advertem a urgência de adoção de medidas perenes de proteção social como atitude política para que a população brasileira não decline vertiginosamente em sua qualidade de vida e bem-estar, com riscos para as atuais e futuras gerações.

E esta é uma mensagem da promoção da saúde, ao se posicionar na defesa da vida, do ecossistema, na desnaturalização das desigualdades e integração de políticas para a potencialização de ações mais includentes e dirigidas, sobretudo para a população mais vulnerável. Isso posto, remete-se aos princípios assumidos pela PNPS: equidade, participação social, autonomia, empoderamento, intersetorialidade, intrassetorialidade, sustentabilidade, integralidade e territorialidade ${ }^{4}$. Esses princípios são âncoras para políticas públicas mais efetivas, justas e igualitárias, com potencial responsivo ao enfrentamento das desigualdades estruturais presentes na sociedade brasileira.

Estudos alertam para a acentuação das desigualdades no período pós-pandemia de covid-19. No contexto nacional, esse alerta ganha mais força diante da combinação de crises: econômica, sanitária e política. Além disso, é necessário reconhecer a pobreza estrutural que existe no Brasil alinhada à alta concentração de renda em um pequeno grupo. 0 Plano Nacional de Enfrentamento à covid-198 registra o desafio de busca de soluções integradoras diante dos problemas complexos desencadeados/agravados pela pandemia e visualiza a intertransdisciplinaridade e participação coletiva como vias de superação. Portanto, é mister considerar os princípios aqui registrados na estruturação de políticas públicas que visem uma adequada proteção social com vistas à superação das desigualdades e respeito às singularidades.

0 foco no território, na comunidade, nas pessoas, permite reconhecer que as vulnerabilidades sociais estão mais presentes nas periferias geográficas e/ou sociais e, nesta senda, dirigir o apoio às populações mais vulneráveis e assegurar a equidade. Mas é preciso atentar-se para que esse agir não reforce as desigualdades; desse modo, é importante lembrar que determinadas formas de cuidar e comunicar podem ser excludentes. No curso da pandemia, podese até mencionar que não faltam informações sobre medidas protetivas, mas como essas informações são traduzidas de modo a alcançar diferentes comunidades, diferentes territórios? Como se dá a tradução do conhecimento? Com quem e como compartilhamos nossos saberes? Como a população tem participado?

Estratégias de comunicação em saúde para mobilizar as comunidades à participação mais ativa requer incorporação de diversas linguagens e abordagens de temas que percorram, no escopo da pandemia, tanto medidas de prevenção do contato com o vírus quanto o aumento da resiliência individual e social. É imperativo ampliar as formas de tradução do conhecimento e reconhecer que desigualdades sinalizam para a não uniformização de ações. Ampliar as linguagens é perpassar pelas diversas atividades humanas, imaginativas, emotivas, lógicas ou científicas, conforme já anunciado no Plano Nacional de Enfrentamento à covid $-19^{8}$.

Quando as pessoas entendem a dinâmica do contágio, as vias de transmissão, as medidas adotadas e seus benefícios, tendem a aumentar a adesão e o sentimento de copartícipes e, dessa forma, lidar melhor com a situação. Assim, a abordagem orientada pela comunicação comunitária é necessária para favorecer a escuta e a vocalização de todos, envolvendo-os no planejamento, na 
execução, reconhecendo os resultados e impactos. A comunicação comunitária baseia-se nos princípios da comunicação popular e caracteriza-se por trabalhar de forma orgânica em âmbito local, mobilizando a participação na transformação do cotidiano das comunidades e grupos populares ${ }^{11}$.

Uma outra situação que tem permeado a pandemia é o distanciamento físico, apregoado como "social", com consequente diminuição de contato e manifestação de afetos físicos, gerando em muitos problemas relacionados à saúde mental. Implantação de intervenções direcionadas para reduzir as situações estressoras advindas ou agravadas por este distanciamento são necessárias. Entende-se que uma rede de apoio socioprofissional se constitui em estratégia importante para a efetivação da atenção oportuna e resolutiva, particularmente quando se consideram as vozes da periferia, historicamente caladas pelo capitalismo. Dito isso, são requeridos princípios, tais como a intersetorialidade e a interprofissionalidade para a superação da fragmentação do cuidado e para que seja assegurada a equidade.

A intersetorialidade e a interprofissionalidade são importantes aliadas no desenvolvimento de ações que impactem positivamente nas condições de vida das pessoas e das comunidades; oportunizam articulação de saberes e vivências, induzem práticas colaborativas, criação de canais para a intervenção nas situações-problema que afligem as comunidades e ainda têm maiores chances de obter resultados satisfatórios e sustentáveis em esfera local ${ }^{12,13}$. 0 exposto reforça a perspectiva de que a Promoção da Saúde não se constitui fim em si mesma, mas fundamentalmente se faz presente nos meios, mediada por seus valores e princípios, e isso inclui a forma como as ações são planejadas e executadas ${ }^{14}$.

A incorporação do conjunto dos princípios aqui mencionados na orientação de políticas e práticas convergirão para que as pessoas se desenvolvam com maior autonomia e participação nos seus modos de viver e promover sua saúde, colaborando para que estas experiências sejam mais sustentáveis e, consequentemente, produzam respostas mais perenes e equânimes para a redução das desigualdades e efetivação de respostas do Estado e da sociedade.

\section{CAMINHOS A SEGUIR: CONSIDERAÇÕES FINAIS}

A crise social, política, econômica e sanitária desnudada pela covid-19 realça a necessidade de fortalecimento de sistemas públicos. No que concerne à saúde e ao Estado brasileiro, é requerido o fortalecimento do SUS como sistema público e universal de saúde, com base no reconhecimento da saúde como direito de cidadania, e integrado a outras políticas econômicas e sociais.

Nesta senda, é razoável reconhecer que o campo da Promoção da Saúde apresenta acúmulo com consistência teórica e metodológica para contribuir nas respostas ao enfrentamento da pandemia de covid-19. Estratégias estas que visem assegurar a garantia da vida e dos direitos, a superação das desigualdades, ampliação de cooperações inter e intrasetoriais, comunicação efetiva com a comunidade e trabalho em rede, movidos pelos sentidos e expressões da solidariedade, da justiça e da coesão social como experiências que permitam a (re)criação e ampliação do espaço social.

Adicionalmente, defende-se uma possivel materialidade dos princípios e valores da Promoção da Saúde, convertendo-os, analogamente, em potente caixa de ferramentas para a tomada de decisão, que pode ser acionada no contexto imediato e mediato à pandemia, reconhecendo, entretanto, que a doença e seu entorno transpõem barreiras geográficas e métricas locais, haja vista que, apesar desta "universalidade", viver o fenômeno da covid-19 é experiência singular, portanto, faz-se necessário pensar global e agir de forma situada.

A pandemia deixará fortes marcações. Transformálas em vetores de mudança de valores e práticas pode ser uma oportunidade de sustentarmos a defesa e a prática da Promoção da Saúde.

\section{CONTRIBUIÇÃO DA AUTORA}

Maria Socorro de Araújo Dias contribuiu na concepção, delineamento, análise e interpretação dos dados, redação e revisão final do manuscrito.

\section{REFERÊNCIAS}

1. Saboga-Nunes L, Levin-Zamir D, Bittlingmayer $U$, Contu P, Oinheiro P, Ivassenko V, et al. A Health Promotion Focus on COVID-19: Keep the Trojan horse out of our health systems. Promote health for ALL in times of crisis and beyond! EUPHA-HP, IUHPE, UNESCO Chair Global Health \& Education [document on the internet]; 2020 [cited 2020 Aug 12]. Available from: https://www.iuhpe.org/index. php/en/iuhpenews/1366-covid19-health-promo 
2. Buss PM. A pandemia e a cooperação internacional em saúde. Blog da Saúde [home page on the internet]; 2020 [cited 2020 Aug 12]. Disponivel em: https://bioeticaediplomacia.org/apandemia-e-a-cooperacao-internacional-em-saude/

3. Brasil. Política Nacional de Promoção da Saúde. Brasília (DF): Ministério da Saúde; 2006.

4. Brasil. Política Nacional de Promoção da Saúde PNaPS: revisão da Portaria MS/GM n ${ }^{\circ} 687$, de 30 de março de 2006. Brasília (DF): Ministério da Saúde; 2014.

5. Ahmed F, Ahmed N, Pissarides C, Stiglitz J. Why inequality could spread COVID-19. Lancet [serial on the internet]. Apr 2020 [cited 2020 Aug 12]; 5. Available from: www.thelancet.com/public-health

6. Brasil. Ministério da Saúde [homepage on the internet]. Painel Coronavírus [cited 2020 Sept 23]. Available from: https://covid.saude.gov.br/

7. Abrams EM, Szefler SJ. COVID-19 and the impact of social determinants of health. Lancet [serial on the internet]. July 2020 [cited 2020 Aug 12]; 8. Available from: https://www.thelancet.com/ journals/lanres/article/PIIS2213-2600(20)30234-4/ fulltext\#back-bib1

8. Frente Pela Vida [homepage on the internet]. Plano Nacional de Enfrentamento da Covid-19 [cited 2020 Aug 18]; 2020. Available from: https://frentepelavida.org.br/uploads/ documentos/PEP-COVID-19 $v 301012$ 20.pdf

9. Ágora Abrasco [homepage on the internet]. As contribuições do campo da Promoção da Saúde para o enfrentamento da pandemia [cited 2020 Aug 17]; 2020. Available from: https://www. abrasco.org.br/site/noticias/agora-abrasco-ascontribuicoes-do-campo-da-promocao-da-saudepara-o-enfrentamento-da-pandemia/49559/

10. Buss PM, Belfort Jr., editorial. Epidemia pela Covid-19: em tempos heterodoxos, medidas heterodoxas. An Acad Nac Med. 2020; 191(2):7-11.

11. Zanchett SA. Reflexões sobre comunicação popular e comunitária: Uma análise a partir das estratégias de comunicação de CPT/MS. Rev Passagens [serial on the internet]. 2015 [cited 2020 Sept 22]; 6(2):108124. Available from: http://www.repositorio.ufc.br/ handle/riufc/46155

12. Dias MSA, Parente JRF, Vasconcelos MI0, Dias FAC. Intersetorialidade e ESF: tudo ou quase nada a ver? Ciênc Saúde Colet. 2014; 19(11). doi: https:// doi.org/10.1590/1413-812320141911.11442014
13. Peduzzi M, Agreli HLF, Silva JAM, Souza HS. Trabalho em equipe: uma revisita ao conceito e seus desdobramentos no trabalho interprofissional. Trab educ saúde [online]. 2020; 18(suppl. 1). doi: https://doi.org/10.1590/1981-7746-sol00246

14. Dias MSA, Oliveira IP, Silva LMS, Vasconcelos MI0, Machado AFAS, Forte FDS, et al. Política Nacional de Promoção da Saúde: um estudo de avaliabilidade em uma região de saúde no Brasil. Ciênc Saúde Colet [serial on the internet]. Jan 2018 [cited 2020 Sept 25]; 23(1):103-114. Available from: http:// www.scielo.br/scielo.php?script $=$ sci arttext\&pid $=$ $\underline{\mathrm{S} 1413-81232018000100103 \& \operatorname{lng}=e n \& \mathrm{nrm}=\text { iso }}$
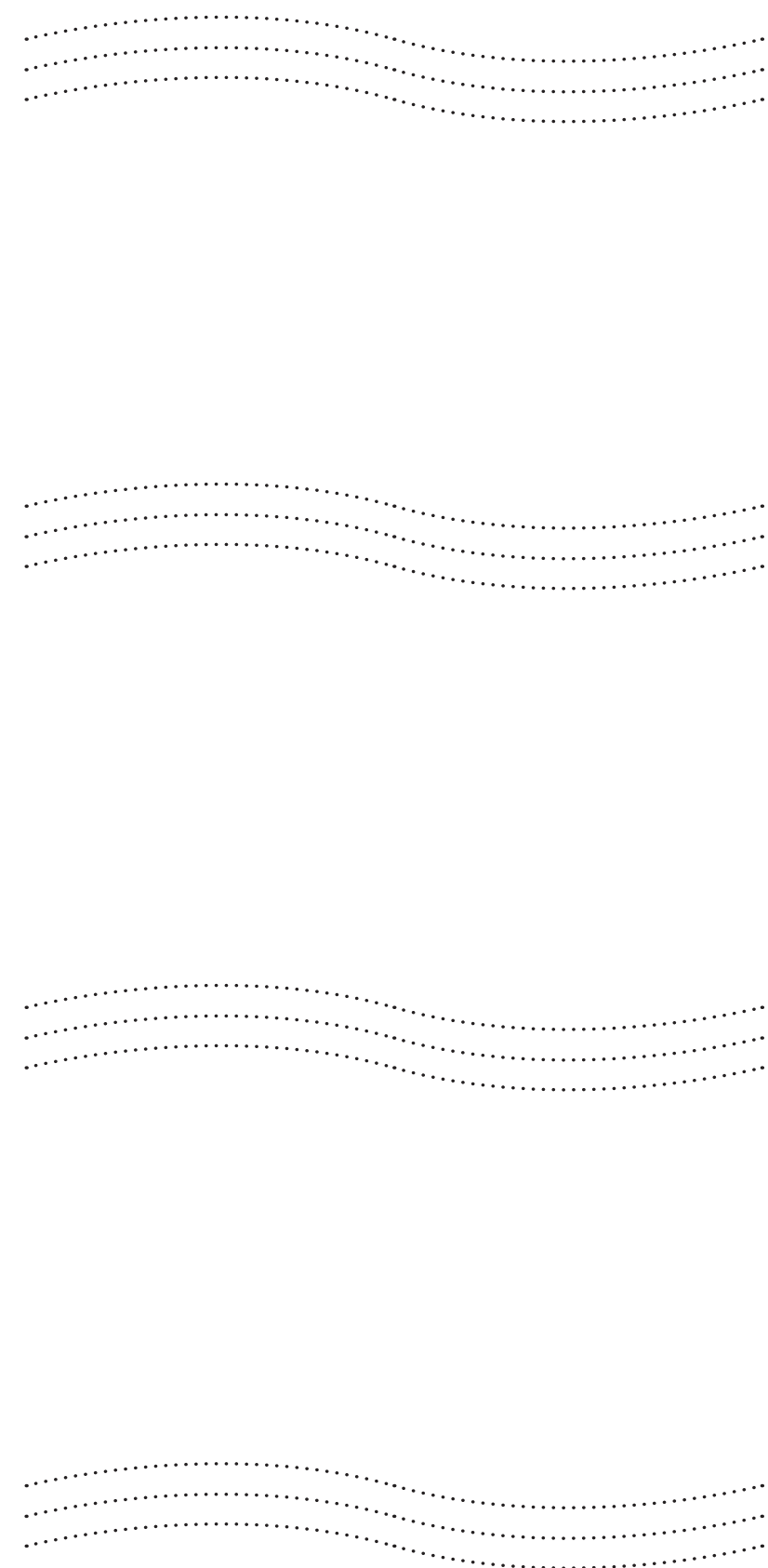